Check for updates

Cite this: RSC Adv., 2017, 7, 37055

\title{
Influence of molecular interplay on the HPAM/UR rheological properties in an aqueous solution
}

\author{
Shuwei Cai, ${ }^{a}$ Hongguo Zhao, ${ }^{b}$ Tianxiang Li, ${ }^{a}$ Xianru He, (D) *a Xin Wang, ${ }^{* a}$ \\ Alisson M. Rodrigues ${ }^{c}$ and Rui Zhang $\mathbb{D}^{d}$
}

Herein, the interaction between partially hydrolyzed polyacrylamide (HPAM) and urea (UR) in an aqueous solution was characterized via differential scanning calorimetry (DSC) and two-dimension correlation spectra (2DCOS). The rheological properties of the HPAM solution (2000 and $2500 \mathrm{mg} \mathrm{L}^{-1}$ ) were studied as a function of the addition of the UR content $\left(50,100,200,300\right.$, and $\left.400 \mathrm{mg} \mathrm{L}^{-1}\right)$. The results indicate that the HPAM/UR solution exhibits higher intrinsic viscosity, apparent viscosity, first normal stress difference, and modulus than the HPAM solution due to the larger hydraulic size of HPAM in the aqueous solution. From the DSC and 2DCOS results, a stronger hydrogen bonding interaction between carbonyl and amino groups was observed in the vicinity of $45^{\circ} \mathrm{C}$. Dynamic light scattering (DLS) was employed to directly analyze the hydrodynamic diameter, and it further confirmed that a certain amount of UR enabled the blending solution to increase the hydraulic size. Moreover, an interplay model was put forward based on the rheology and dynamic light scattering data.

Received 9th May 2017

Accepted 28th June 2017

DOI: $10.1039 / \mathrm{c} 7 \mathrm{ra05263d}$

rsc.li/rsc-advances

heat treatment, respectively. Rheological properties of the

\section{Introduction}

As a type of polymer flooding agent, partially hydrolyzed polyacrylamide (HPAM) has received significant interest due to its applications in tertiary oil recovery, especially in reducing liquid permeability, increasing sweep efficiency or microscopic oildisplacing efficiency, and some other aspects. ${ }^{1-3}$ It is vital to the mechanism of polymer flooding for enhanced oil recovery that the polymer flooding agents exhibit a relatively higher viscoelasticity performance following injection..$^{4-6}$ However, viscoelasticity of the HPAM solution is strongly influenced by shear stress, electrolyte, temperature, and $\mathrm{pH}$ due to its weak polyelectrolyte structure. ${ }^{7-11}$ HPAM exhibits weak polyelectrolyte behavior in the aqueous solution. Mamdouh T. Ghannam ${ }^{12}$ have found that the addition of $\mathrm{NaCl}$ to the PAM solution decreases the apparent viscosity of the solution. Zhang Qing et al. ${ }^{13}$ found that the shielding effect of inorganic salts enables the HPAM coil to transform into a more constructive conformation in a saline solution. However, this effect tended to decline at high salt concentrations $\left(C>0.2 \mathrm{~mol} \mathrm{~L}^{-1}\right) .{ }^{7}$ On the other hand, the HPAM solution easily shows shear thinning and viscosity break properties once it suffers from shear stress and

${ }^{a}$ School of Materials Science and Engineering, State Key Laboratory of Oil and Gas Reservior Geology and Exploitation, Southwest Petroleum University, ChengDu, China 610500. E-mail: xrhe@swpu.edu.cn

${ }^{b}$ Petrochemical Research Institute, PetroChina, Lanzhou, China 730060

${ }^{c}$ Vitreous Materials Laboratory, Department of Materials Engineering, Federal University of São Carlos, UFSCar, 13.565-905, São Carlos, Brazil

${ }^{d}$ Institut für Physik, Universität Rostock, Albert-Einstein-Str. 23-24, Rostock, Germany 18051 solution refer to the polymer structure, component, conformation, and macromolecular interplay. Through molecular interplay, the alternation of polymer conformation and improvement of rheological behavior can be realized. Several research groups have developed approaches to solve these issues. As a widely investigated example, surfactants were extensively utilized for improving salt tolerance and temperature resistance. ${ }^{14-20}$ Although these observations were particularly helpful for holding solution viscosity, the crude oil exploited through injecting polymer/surfactant flooding inevitably contained a certain amount of surfactant and presented difficulties during post-processing owing to the unique surface activity of the surfactant. ${ }^{21}$ To enhance the oil recovery efficiency and lower the cost, inexpensive and well-separated modifiers can be employed.

Urea is a type of salt that consists of a carbonyl and two polar amino groups. It is also utilized as a fertilizer in agricultural production ascribing to its low-cost and favorable effect. More importantly, UR is easily separated from oil-water blend solutions. S. A. Frangou ${ }^{10}$ have explored the effect of urea on the chain conformation of xanthan solution and found that the concentration of urea has a noticeable influence on the conformation transformation of xanthan. Moreover, several types of studies on the thermal cross-linking of carboxylate/ amine groups have already been reported and suggested the potential for temperature resistance..$^{22,23}$ In this study, the rheological properties of HPAM/UR solutions were explored via viscosimetry and rheometry using different detection models and temperatures. Dynamic light scattering was employed to 
directly analyze the hydrodynamic diameter. ${ }^{24-32}$ DSC and 2DCOS techniques were carried out to obtain further evidence of the interplay between HPAM segments and urea molecules under variable temperature conditions from a different perspective. ${ }^{33-38}$

\section{Experimental}

\section{Materials}

HPAM (industrial grade) was supplied by the Shengli Oil Field, China. The weight-average molecular weight of HPAM, $2.3 \times$ $10^{7} \mathrm{~g} \mathrm{~mol}^{-1}$, was determined via static light scattering tests, and the hydrolysis degree (HD) was $27 \%$. Samples were employed after further purification. Urea and $\mathrm{NaCl}$ were purchased from Chengdu Kelong Chemical Agents Company (AR grade). Homemade ultrapure water was used in the preparation of solutions.

The samples for rheological measurements were prepared by dissolving HPAM powder in ultrapure water and then precipitating with methanol. The precipitate was washed and placed in a vacuum drying box at $35^{\circ} \mathrm{C}$ for $24 \mathrm{~h}$, and the dried product was used for mother solution preparation. All the samples were obtained by the dilution method, and dilution solvents were sodium chloride solution and $\mathrm{NaCl} / \mathrm{UR}$ solution. The concentration of the sodium chloride solution is $0.1 \mathrm{~mol} \mathrm{~L}^{-1}$. Samples for the DSL tests were diluted to $50 \mathrm{mg} \mathrm{L}^{-1}$ and centrifuged (12 $000 \mathrm{rpm}$ ) for about $10 \mathrm{~min}$. Before being placed in the sample cell, the test solutions were filtered via a $0.22 \mu \mathrm{m}$ microporous filter. For the FT-IR and DSC experiments, the concentration was fixed at $10 \mathrm{wt} \%$. All the testing samples were aged for 12 hours at ambient temperature before use.

\section{Instruments and measurements}

The intrinsic viscosity measurements were carried out using an Ubbelohde viscometer (globule volume was $4 \mathrm{~mL}$ and the average capillary diameter was $0.65 \mathrm{~mm}$ ) in a water bath with automatic temperature control. The temperature was controlled at $25 \pm 0.1^{\circ} \mathrm{C}$. In all the cases, the flow time of the solvent $(\mathrm{NaCl}$ solution) was less than 100 seconds such that kinetic energy corrections were made. The corresponding Ubbelohde viscometer was calibrated via calibration experiments. The corresponding parameters of the standard solutions employed are listed in Table 1.

All the reduced viscosities were calculated by eqn (1) as follows:

$$
\eta_{\mathrm{r}}=(A t-B t) /\left(A t_{\mathrm{s}}-B t_{\mathrm{s}}\right)
$$

Table 1 Density and viscosity of the standard solutions

\begin{tabular}{lll}
\hline & Acetone; $T=25{ }^{\circ} \mathrm{C}$ & $n$-Butanol; $T=25{ }^{\circ} \mathrm{C}$ \\
\hline$\rho\left(\mathrm{g} \mathrm{mL}^{-1}\right)$ & 0.7851 & 0.8051 \\
$\eta_{\mathrm{r}(\mathrm{Pa} \mathrm{S})}$ & $3.075 \times 10^{-3}$ & $2.6390 \times 10^{-2}$
\end{tabular}

where $\eta_{\mathrm{r}}$ is the relative viscosity of the solution, which is the ratio of the viscosity of the polymer solution to that of neat solvent; $t$ is the efflux time of the calibrating liquid; and $t_{\mathrm{s}}$ is the efflux time of the solvent. The calibration parameters are $A=$ 0.00016010212 and $B=0.01211235447$. For rheological measurements, the AR-2000 rheometer (TA Instrument, America) was employed with a standard parallel plate (diameter $=40$ $\mathrm{mm}$ ). The gap was set at $200 \mu \mathrm{m}$, and the middle-temperature error was controlled at less than $0.1{ }^{\circ} \mathrm{C}$. To cope with various test requirements, flow sweep and oscillation models were selected in the applicable shear rate or angular frequency. The first normal stress difference was measured by the Anton Paar MCR 302 rheometer (Anton Paar, America). Dynamic light scattering measurements were conducted using a Brookhaven Instrument (BI-200SM) at $25{ }^{\circ} \mathrm{C}$. All time-resolved FTIR spectra were obtained using a resolution of $2 \mathrm{~cm}^{-1}$ via a NICOLET-6700 infrared spectrometer equipped with an electronic cell. HPAM sample was sealed between two $\mathrm{CaF}_{2}$ tables. In these tests, 64 scans were available for an acceptable signal-to-noise ratio. Manual method was used to control the temperature at the rate of ca. $0.5{ }^{\circ} \mathrm{C} \mathrm{min}^{-1}$ with an increment of $5{ }^{\circ} \mathrm{C}$. The baseline correction process was performed by the software Omnic ver. 8.2.0.387. $2 \mathrm{D}$ correlation analysis was conducted by the software 2D shige ver. 1.3 (2D-shige (c) Shigeaki Morita, Kwansei-Gakuin University, Japan, 2004-2005) and further plotted into contour maps via the Origin 8.0. Calorimetric measurements were performed using a Mettler Toledo DSC823-TGA/SDTA85/e thermal analyzer at the rate of $1{ }^{\circ} \mathrm{C} \mathrm{min}^{-1}$ during the heating process.

\section{Results and discussion}

\section{Effect of UR on the viscosity of HPAM}

It is known that hydrodynamic size is closely related to the rheological behavior of the polymer dilute solution, and according to the polymer solution theory, intrinsic viscosity $([\eta])$ can reflect the variation of hydrodynamic size and molecular conformation of a polymer. Thus, Fig. 1 shows the effect of UR addition on the intrinsic viscosity of the HPAM solution at $T=$ $25{ }^{\circ} \mathrm{C}$. It is observed that the intrinsic viscosity increases with

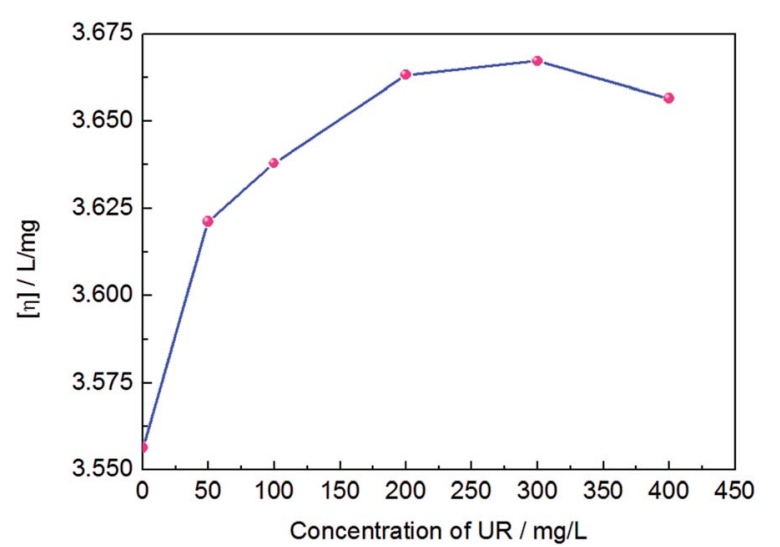

Fig. 1 Intrinsic viscosity versus the UR concentration for the HPAM solution $\left(T=25^{\circ} \mathrm{C}\right)$. 
UR addition and reaches maximum at $300 \mathrm{mg} \mathrm{L}^{-1}$ of the UR content. This behavior is a result of an interplay between HPAM segments and UR molecules as well as acylamino and amino interactions that are manifested by variation in the UR content. Moreover, changes in $[\eta]$ suggest that the alternation of the HPAM coil conformation occurs in the aqueous solution. The UR molecules enable HPAM coils to be more extensive and increase the hydrodynamic radii to some degree. Therefore, a certain amount of UR is especially useful for the improvement of viscosity.

Fig. 2 shows the effects of UR addition on the apparent viscosity in $2000 \mathrm{mg} \mathrm{L}^{-1}$ of the HPAM solutions at $25{ }^{\circ} \mathrm{C}$. It was notable that in the low shear rate area (less than $0.3 \mathrm{~S}^{-1}$ ), similar trends were observed for the apparent viscosity of HPAM. In the HPAM/UR solutions, the viscosity increases in the order of UR concentration: $0 \mathrm{mg} \mathrm{L}^{-1}<50 \mathrm{mg} \mathrm{L}^{-1}<100 \mathrm{mg} \mathrm{L}^{-1}<400 \mathrm{mg} \mathrm{L}^{-1}$ $<200 \mathrm{mg} \mathrm{L}^{-1}<300 \mathrm{mg} \mathrm{L}^{-1}$, and the maximum in these cases occurred at $300 \mathrm{mg} \mathrm{L}^{-1}$. However, the viscosities of the solutions, which are close to each other in the high shear rate area, are expected to result in the complete orientation of macromolecules in the aqueous solution. When a higher shear rate is imposed, the motion of the chain segment play as the main migration unit, which enables molecule conformation to vary and causes a decrease in the hydrodynamic volume. Moreover, shear stress can damage hydrogen bonding between HPAM and UR, especially at higher shear rates. Consequently, the viscosity of the solution reduces.

The apparent viscosities for variable HPAM concentrations were measured to explore the effect of HPAM concentration on the solution viscosity in the HPAM/UR system. Fig. 3 shows the apparent viscosity versus shear rate curves for different HPAM solutions involving blends formed from two compositions: HPAM and $300 \mathrm{mg} \mathrm{L}^{-1} \mathrm{UR}$. The deviation of apparent viscosity for $2000 \mathrm{mg} \mathrm{L}^{-1}$ and $2500 \mathrm{mg} \mathrm{L}^{-1}$ HPAM solution in Fig. 3 is most likely a consequence of the effects of HPAM concentration and hydrodynamic volume at lower shear rates. For the $2000 \mathrm{mg}$ $\mathrm{L}^{-1} \mathrm{HPAM} / 300 \mathrm{mg} \mathrm{L}^{-1}$ UR solution, the molar ratio of UR and HPAM is bigger than the ratio in the $2500 \mathrm{mg} \mathrm{L}^{-1} \mathrm{HPAM} / 300 \mathrm{mg}$

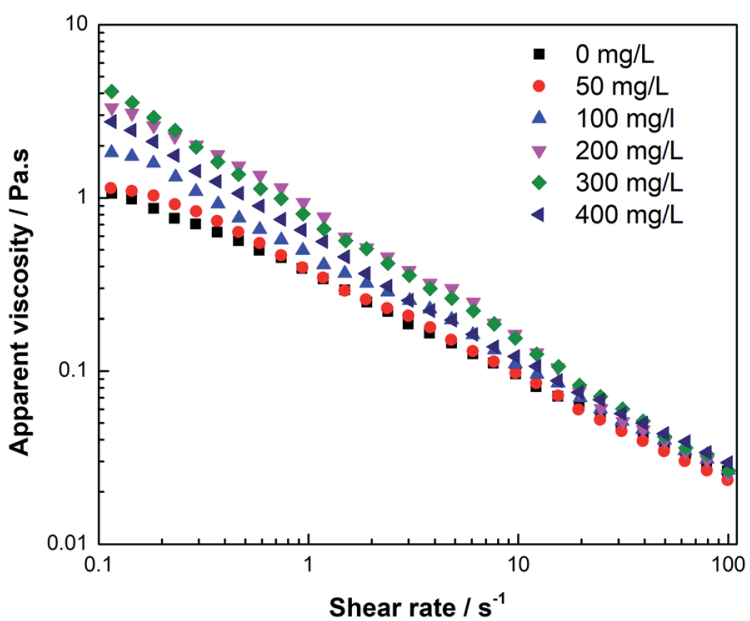

Fig. 2 Apparent viscosity of the $2000 \mathrm{mg} \mathrm{L}^{-1}$ HPAM solutions versus the shear rate for various UR contents $\left(T=25^{\circ} \mathrm{C}\right)$.

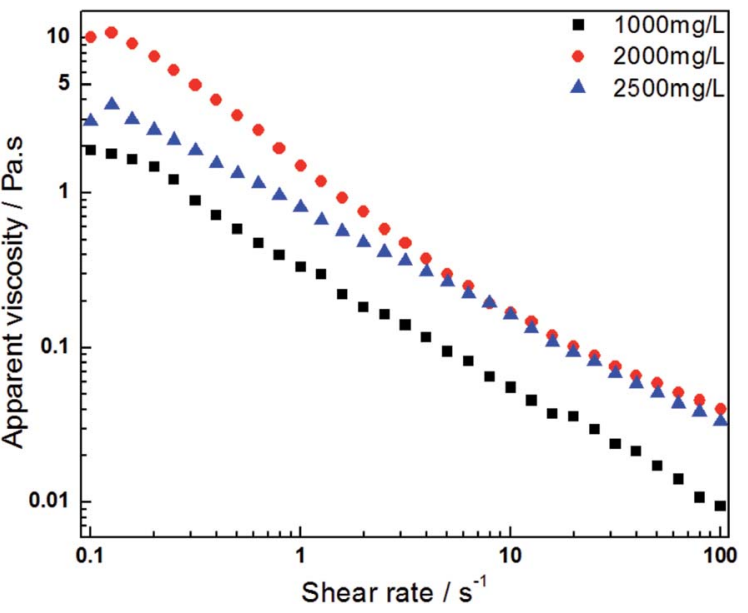

Fig. 3 Apparent viscosity of the HPAM/UR (300 $\mathrm{mg} \mathrm{L}^{-1}$ UR) blend solution versus the shear rate for various HPAM concentrations ( $T=$ $\left.25^{\circ} \mathrm{C}\right)$.

$\mathrm{L}^{-1}$ UR solution. Therefore, the interplay between HPAM and UR is more prominent and forms a larger hydrodynamic volume in the former solution. To a certain degree, it confirms that there is an interaction between the UR and HPAM structural unit. It also provides evidence that UR has a significant influence on solution viscosity in this system. As abovementioned, the orientation of the HPAM molecule is completed when the HPAM/UR solution suffers from high shear rates. Thus, the hydrodynamic volume of polymer is no longer the decisive factor for viscosity. However, the concentration of the polymer has a greater effect in this case. As a result, the viscosities of the two abovementioned systems are almost the same when they suffer from high shear rates. The viscosity of the $1000 \mathrm{mg} \mathrm{L}^{-1}$ HPAM/300 $\mathrm{mg} \mathrm{L}^{-1}$ UR solution, however, is lower than that of the remaining two solutions under the present experimental condition; this indicates that the variation of the HPAM concentration is the dominant factor for the apparent viscosity.

Based on the experimental results shown in Fig. 1-3, a proper amount of UR in the HPAM solution effectively enhances the apparent viscosity, but is less efficient when excess UR is added. The possible reason is that the HPAM molecular conformation transforms and possesses bigger hydrodynamic volume; this is caused by hydrogen bonds formed by the amino groups of UR and the HPAM structural unit, which involves molecular interaction in progress. The specific interplay is described as the adjacent carbonyls along the HPAM chain adsorbed by UR via hydrogen bonding. Thus, the HPAM chain segments become rigid and increase the hydraulic size. However, this influence is finite. At low UR contents, the specific interaction holds the dominated position, which has been manifested by the enhancement of the intrinsic viscosity of HAPM/UR. In contrast, a high UR content may cause a reduction in the intrinsic viscosity.

When the concentration of UR was lower than $300 \mathrm{ppm}$, the interaction between the side groups of HPAM and UR was weak owing to lesser number of UR molecules in the solution. However, when the UR content exceeded 300 ppm, the increase 
in the interaction enabled the hydraulic size of the HPAM molecules to enlarge, causing the intrinsic viscosity to reach maximum. As the concentration of UR is further increased, the UR molecules might have interaction with two or more side groups in the HPAM molecules, which causes the decrease in intrinsic viscosity. Based on the abovementioned discussion, this interaction is more efficient for the enhancement of intrinsic viscosity under the present experimental conditions when the content of UR is $300 \mathrm{mg} \mathrm{L}^{-1}$. A schematic of the specific interaction in the aqueous solution is presented in Fig. 4.

From another perspective, the HPAM hydraulic size and how closely migration matches angular frequency have a significant influence on the macromolecular motion behavior in blend solution when oscillation stress is imposed. The related parameters obtained from the oscillation measurement model can reflect information about the molecular motion. The data corresponding to the storage modulus and loss modulus for the HPAM and HPAM/UR solutions are plotted in Fig. 5 as a function of angular frequency. Values of $G^{\prime}$ for HPAM/UR, which are expressed by the solid pattern, are higher than those for HPAM. Similar trends for $G^{\prime \prime}$ were observed. The improvement of $G^{\prime}$ and $G^{\prime \prime}$ is due to the enhancement of the deformation resistance caused by an increase of coil size. Hydrodynamic volume, however, has been further discussed hereinafter. Herein, it is notable that the addition of UR can improve the viscoelasticity of the HPAM solution; on the other hand, this also indicates that the interaction between UR and HPAM occurred in the aqueous solution.

DLS was employed to analyze the distribution of the hydrodynamic diameter $\left(D_{\mathrm{h}}\right)$ when the HPAM concentration was diluted to $50 \mathrm{mg} \mathrm{L}^{-1}$. Although the HPAM concentration was diluted, the UR content was maintained at $300 \mathrm{mg} \mathrm{L}^{-1}$. The results shown in Fig. 6 are consistent with the previously proposed rheological properties discussion. The distribution of the hydrodynamic diameter for the HPAM solution mainly concentrated at 110-300 $\mathrm{nm}$, whereas the distribution for the HPAM/UR solution was at 200-700 $\mathrm{nm}$. This is probably

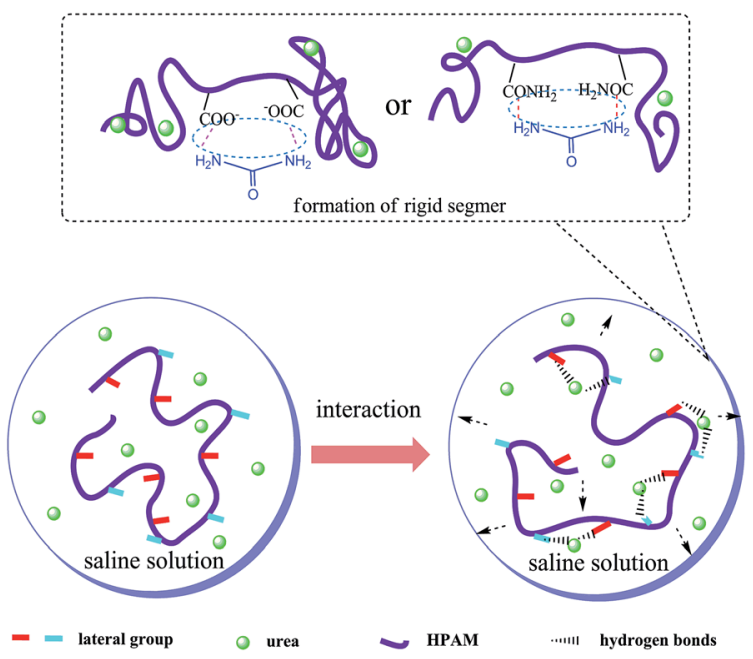

Fig. 4 Schematic for the short-range and long-range interactions.

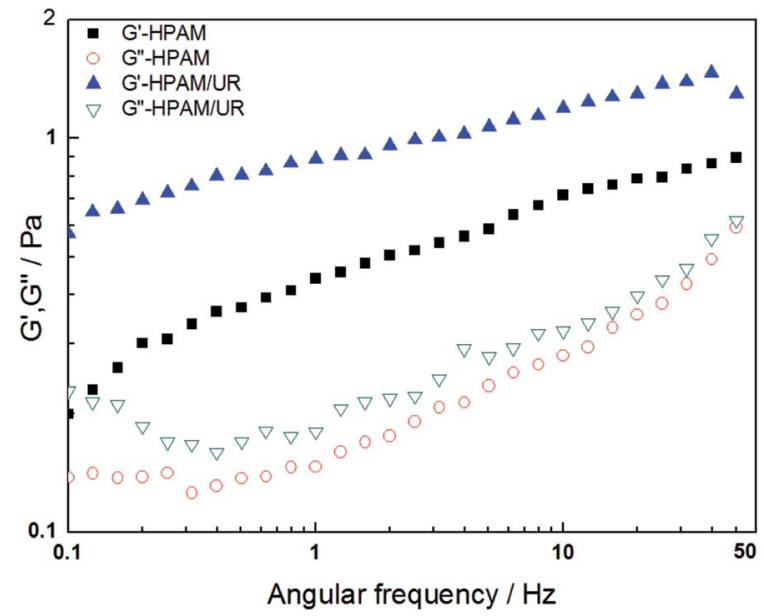

Fig. $5 G^{\prime}$ and $G^{\prime \prime}$ as a function of frequency for the $2000 \mathrm{mg} \mathrm{L}^{-1}$ HPAM and HPAM/UR $\left(300 \mathrm{mg} \mathrm{L}^{-1}\right)$ solutions $\left(T=25^{\circ} \mathrm{C}\right)$.

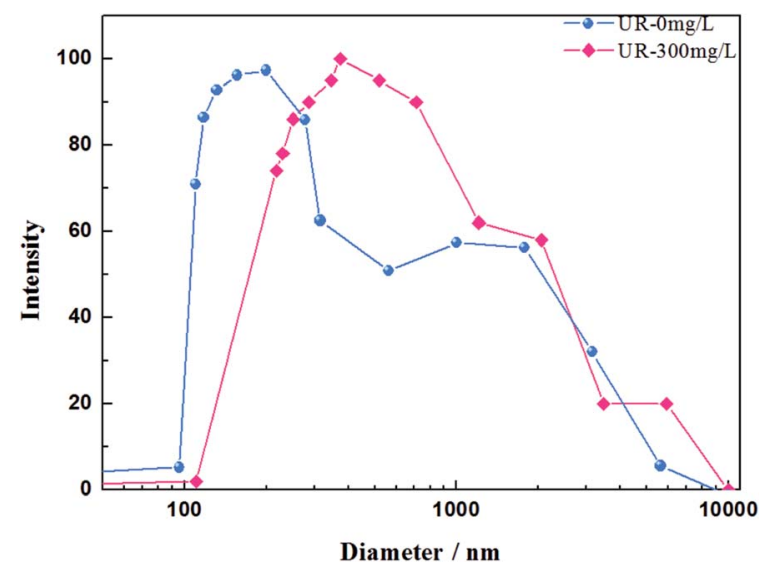

Fig. 6 DLS measurements of the HPAM and HPAM/UR solutions at $25^{\circ} \mathrm{C}$.

a consequence of the interplay between HPAM and UR. Acylamino, which is on the HPAM lateral chain, can bond to UR via hydrogen bonding. According to the analysis of Fig. 4, an extensive conformation and increase of $D_{\mathrm{h}}$ are benefited from the formation of hydrogen bonding when UR was added under the present experimental condition. Thus, the larger the $D_{\mathrm{h}}$, the higher the viscosity of the dilute solution..$^{39,40}$

\section{Effect of temperature on the HPAM/UR interplay and rheological properties}

Temperature has significant effects on the intermolecular interaction and migration. In our previous study, ${ }^{\mathbf{1 1}}$ hydrogen bonding interaction in an aqueous solution explored by DSC differential spectrum was reported, and it was found that in the low shear rate region, the maximum apparent viscosity value occurred near $40{ }^{\circ} \mathrm{C}$ in the temperature-rise period. Fig. 7 shows the apparent viscosity versus the shear rate curves for the HPAM solutions involving blends formed from two different compositions: $2000 \mathrm{mg} \mathrm{L}^{-1}$ HPAM and UR. ${ }^{41}$ In the low shear rate area, 


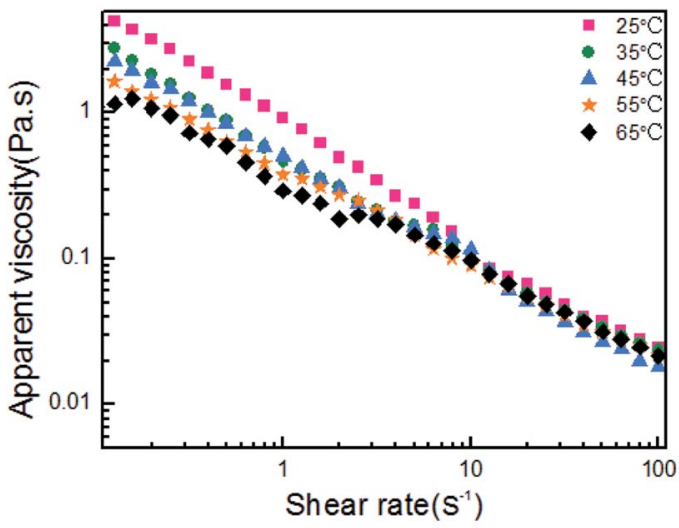

(a)

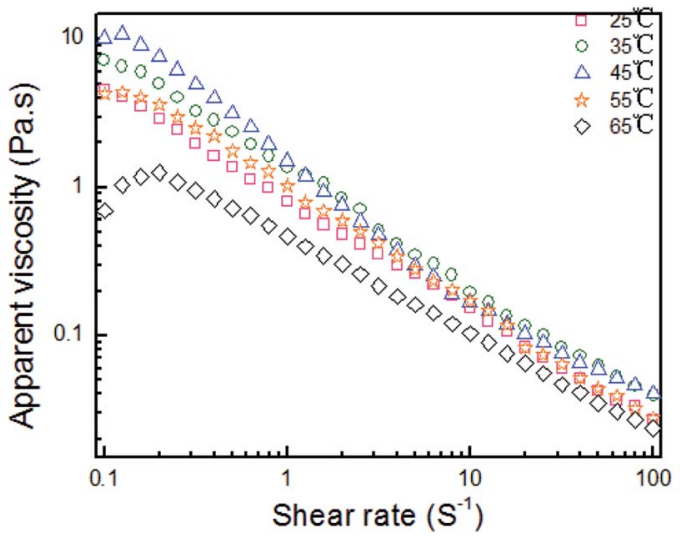

(b)

Fig. 7 (a) Apparent viscosity of $2000 \mathrm{mg} \mathrm{L}^{-1}$ HPAM with the $0 \mathrm{mg} \mathrm{L}^{-1}$ UR content versus shear rate at various temperatures. (b) Apparent viscosity of $2000 \mathrm{mg} \mathrm{L}^{-1}$ HPAM with $300 \mathrm{mg} \mathrm{L}^{-1}$ UR content versus shear rate at various temperatures.

the apparent viscosity reduces with the increasing temperature when the UR content is $0 \mathrm{mg} \mathrm{L}^{-1}$, as shown in Fig. 7(a). While in Fig. 7(b), the viscosity initially increases and then decreases with the increasing temperature in the same shear rate region when the UR content in the blends is $300 \mathrm{mg} \mathrm{L}^{-1}$. In the HPAM solutions, the active ability of the molecular motion unit was improved and manifested via a reduction in the viscosity. However, for various temperature series, the viscosity of the blend solution increases in the order: $65{ }^{\circ} \mathrm{C}<25^{\circ} \mathrm{C}<55^{\circ} \mathrm{C}<$ $35{ }^{\circ} \mathrm{C}<45{ }^{\circ} \mathrm{C}$. It was considered that the addition of UR causes an increase in the viscosity, and this may involve hydrogen bond damage and restructuring, conformation variation, and thermal effect. $^{41}$ This behavior has been discussed further in the following section.

As discussed above, intrinsic viscosity can directly reflect interplay in a dilute solution. The effect of the temperature on $[\eta]$ is shown in Fig. 8. In these tests, two types of solutions were employed to measure the $[\eta]$ : one is the HPAM solution and the other is a blend of HPAM and UR. The UR concentration was maintained at $300 \mathrm{mg} \mathrm{L}^{-1}$ in the blends. The results show that the viscosity of the HPAM/UR solution first increases and then decreases with the increasing temperature, which is in accordance with the changes of the apparent viscosity shown in Fig. 7(b). At $T<30{ }^{\circ} \mathrm{C}$ and $T>45^{\circ} \mathrm{C},[\eta]$ for the HPAM solution is relatively lower than the $[\eta]$ of the blend solution, whereas it is

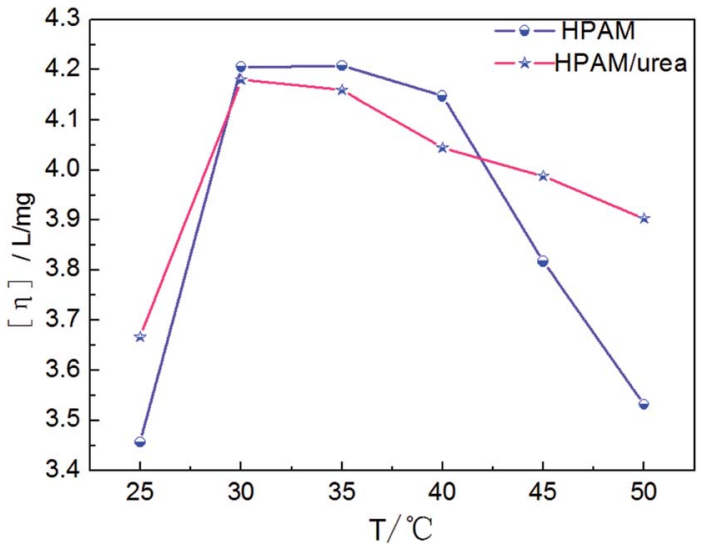

Fig. 8 Temperature dependence of the intrinsic viscosity for the HPAM and HPAM/UR (300 $\mathrm{mg} \mathrm{L}^{-1}$ ) solutions.

contrary in the situation where the temperature ranges from $30{ }^{\circ} \mathrm{C}$ to $45{ }^{\circ} \mathrm{C}$. When the temperature is higher than $40{ }^{\circ} \mathrm{C}$, the downtrend of $[\eta]$ observed in the HPAM/UR solution is gentler than that in the HPAM solution. Partly, the addition of UR can improve the temperature resistance of the HPAM solution. The possible reason may be stated as follows: HPAM exhibits weak polyelectrolyte behavior in an aqueous solution; electrostatic repulsion and solvation have significant effect on its conformation and hydraulic size. For electrostatic repulsion, the higher the polyelectrolyte solution ionization degree, the stronger the repulsive interaction. Thus, the solution has a higher intrinsic viscosity. From another perspective, the effect of solvation in this system is manifested by the hydrogen bonding interaction. At lower temperatures, short-range interplay holds the dominated position, which has been discussed in Fig. 4. Once the temperature increases, the migration ability of the counter-ion becomes stronger, and the ionization intensity increases. Thus, electrostatic repulsion is enhanced, and a higher intrinsic viscosity is observed.

On the other hand, high temperature does not favor the formation of hydrogen bonds and solvation. As a result, the intrinsic viscosity decreases. When solutions are exposed to lower temperatures $\left(T<30{ }^{\circ} \mathrm{C}\right.$ in the present tests), the former holds the dominated position, which was manifested by the enhancement of $[\eta]$ with the increasing temperature, whereas the latter has greater influence when the solutions suffer from higher temperatures $\left(t>35{ }^{\circ} \mathrm{C}\right)$ and the $[\eta]$ decreases with the increasing temperature. It is notable that the $[\eta]$ of the HPAM/ UR system is higher than the $[\eta]$ of the HPAM solution when $45{ }^{\circ} \mathrm{C}<T<50{ }^{\circ} \mathrm{C}$, as shown in Fig. 8. This is probably a consequence of large numbers of the short-range interplay between HPAM and UR within this temperature range. The corresponding evidence has been given in the following section.

The $G^{\prime}$ and $G^{\prime \prime}$ data for the HPAM and HPAM/UR solution are plotted in Fig. 9 as a function of angular frequency. For the temperature influence series, the $G^{\prime}$ and $G^{\prime \prime}$ of the HPAM solution increase in the order $60{ }^{\circ} \mathrm{C}<45{ }^{\circ} \mathrm{C}<35^{\circ} \mathrm{C}$, whereas for the HPAM/UR blend solution, the order is $35{ }^{\circ} \mathrm{C}<60{ }^{\circ} \mathrm{C}<45^{\circ} \mathrm{C}$. As per the previous discussion on modulus, the enhancement of $G^{\prime}$ 

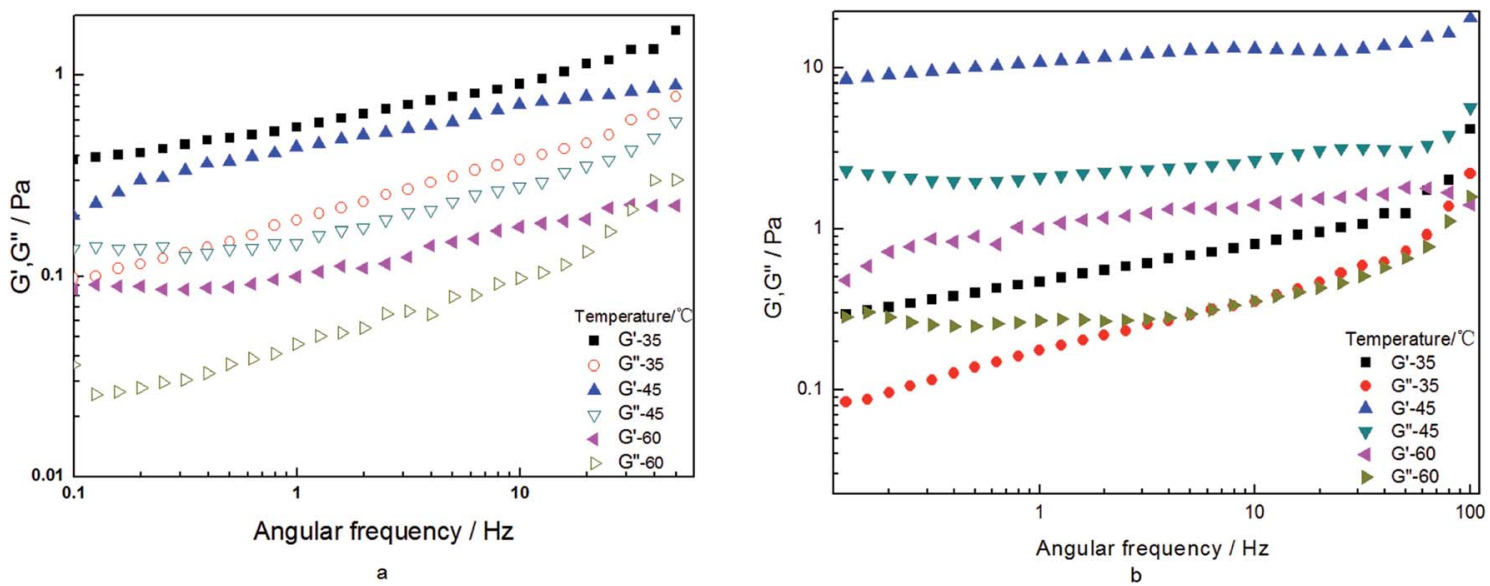

Fig. 9 (a) Modulus of $2000 \mathrm{mg} \mathrm{L}^{-1}$ HPAM. (b) Modulus of $2000 \mathrm{mg} \mathrm{L}^{-1} \mathrm{HPAM} / 300 \mathrm{mg} \mathrm{L}^{-1}$ urea solution.

and $G^{\prime \prime}$ is probably due to the strong, short-range interaction between HPAM and UR at $T=45^{\circ} \mathrm{C}$. Moreover, the hydraulic size has great influence on the elastic properties of the polymer in an aqueous solution. As is known, the first normal stress difference can reflect elastic performance. Compared with a small hydraulic size polymer, a bigger hydraulic size polymer would suffer from greater motion resistance. Therefore, it is easy to yield elastic deformation when shear stress is imposed on a bigger coil-sized macromolecule. The effect of temperature on first normal stress difference for HPAM and HPAM/UR (300 $\mathrm{mg} \mathrm{L}^{-1}$ ) solution is shown in Fig. 10. Contrary to the HPAM solution, higher values of the first normal stress difference were observed in the HPAM/UR system under the experimental condition, especially in high shear rate area. This indicates that the latter exhibited excellent performance when shear stress was imposed. Thus, the results shown in Fig. 10 are consistent with the previous discussion, that is, a larger coil size is formed in the HPAM/UR system due to the short-range interaction.

To further explore the molecular interplay, the calorimetric measurements were carried out to explore the temperature effect on the interaction involving HPAM and UR. First, the TG test was performed to explore whether degradation reactions existed with the increasing temperature. The TG and DTG curves for the $40 \mathrm{wt} \%$ HPAM solution are shown in Fig. 11. An apparent weight loss only occurred at $T=155^{\circ} \mathrm{C}$ in the entire test temperature range. Moreover, a peak appears at the same position in the corresponding DTG curve. This evidence indicated that no degradation reactions occurred when the temperature was less than $155{ }^{\circ} \mathrm{C}$. In a way, in the lower temperature range, the variations of rheological behavior with the increasing temperature are expected to be related to the change in the strength of the molecular interaction. On the basis of abovementioned discussion, DSC differential spectrum was employed to explore the abovementioned interplay in an aqueous solution. ${ }^{41-43}$ The difference of heat flow (DHF) between HPAM/UR and HPAM solutions and the corresponding DSC curves are shown in Fig. 12. Through the difference of heat flows of two types of polymer solutions, thermal effect of the molecular interaction between UR and HPAM stood out, and the energy variations under the increasing temperature conditions were obtained. The red curve in Fig. 12 is the heat flow curve for the HPAM/UR solution, and the blue curve is the heat flow curve for the HPAM solution. The green curve is the
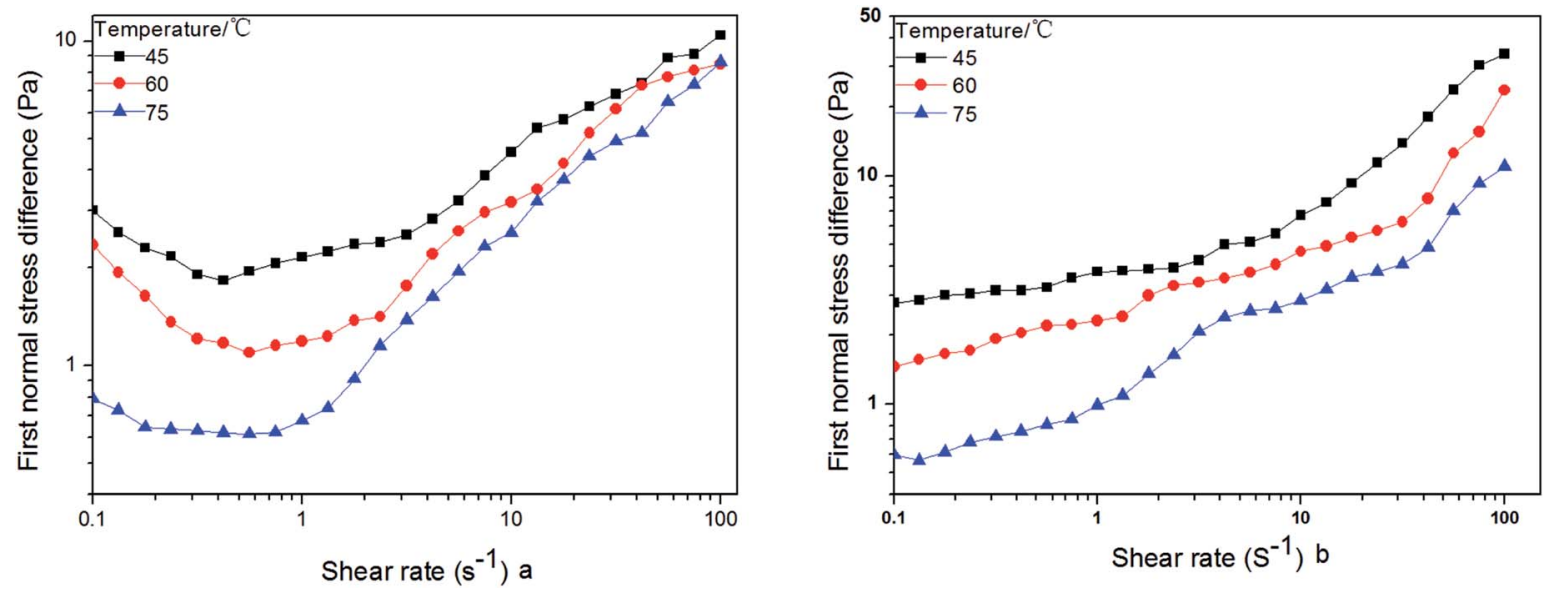

Fig. 10 First normal stress difference for the $2000 \mathrm{mg} \mathrm{L}^{-1}$ HPAM (a) and HPAM/UR solution versus shear rate (b). 

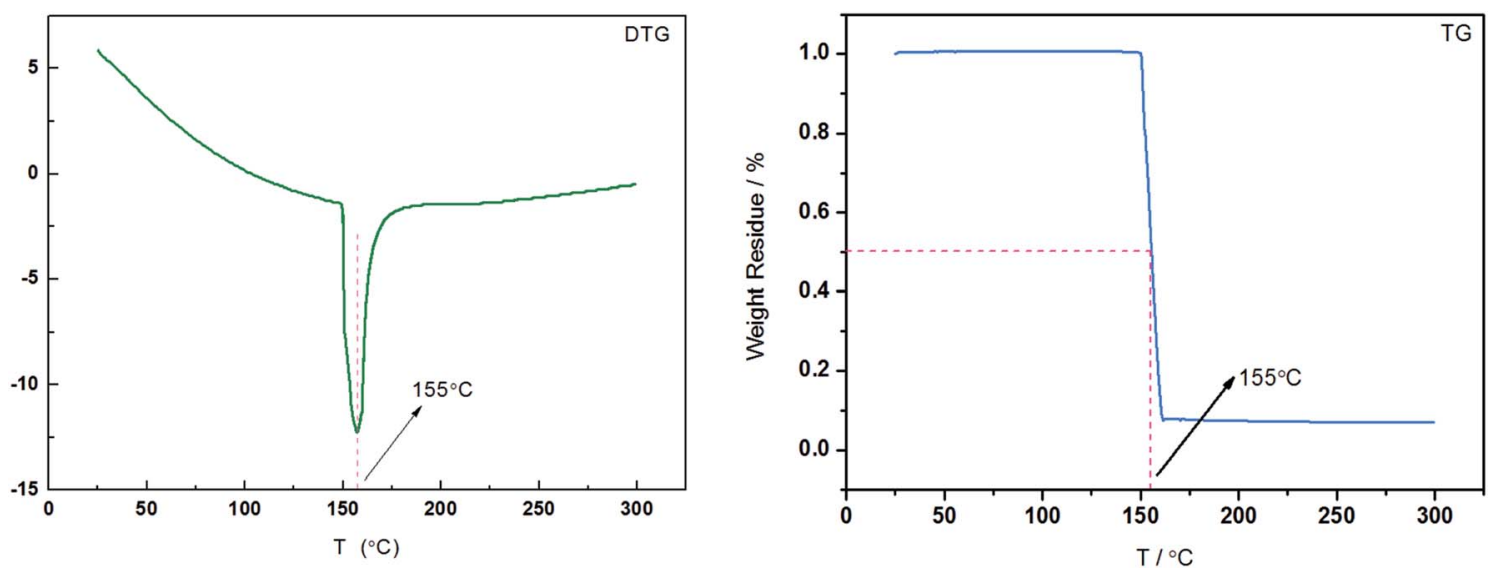

Fig. 11 TG and DTG curves for the 40 wt\% HPAM solution.

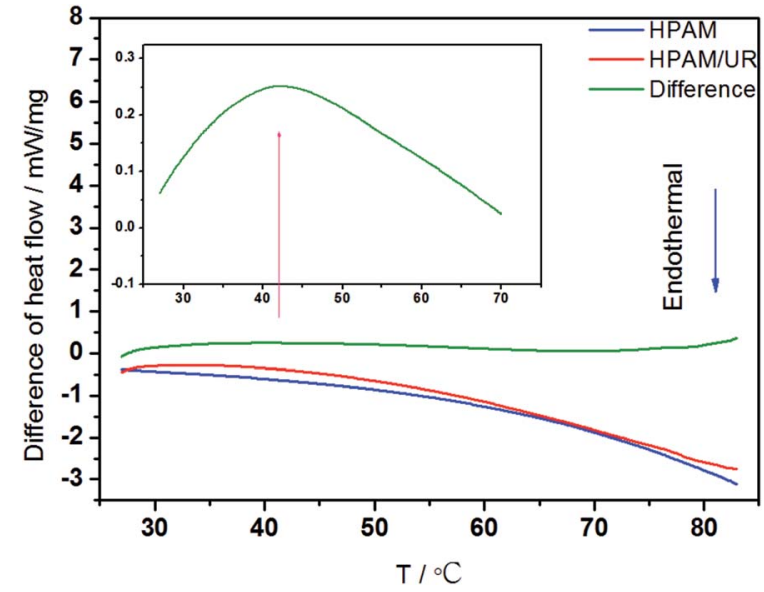

Fig. 12 The difference of heat flow between HPAM/UR and HPAM solutions versus temperature.

abovementioned DHF curve. The inset is a local amplification of the original curve in the temperature range: $27-70{ }^{\circ} \mathrm{C}$. In the entire temperature-rise period, the variations of heat flow for both HPAM and HPAM/UR are not visible. However, a visible exothermic process is observed in the local amplifying figure, and the strongest exothermal process appears near $40{ }^{\circ} \mathrm{C}$. Based on the previous analysis of rheological behavior, it is possible that more hydrogen bonds form between the construction unit of HPAM and UR in this temperature range, and the exothermal process corresponds to the process of hydrogen bond formation.

Two-dimensional correlation spectra (2DCOS) is a mathematical method whose basic principles were first proposed by Noda in 1986. Due to the various responses of different species to external variables, helpful information about molecular interaction or conformation changes can be extracted, which cannot be directly obtained from a conventional 1D spectrum. On the basis of the temperature effect on the rheological properties and temperature region obtained from the DSC and apparent viscosity tests, infrared spectra obtained in the range from $30^{\circ} \mathrm{C}$ to $50^{\circ} \mathrm{C}$ were chosen to perform the 2DCOS analysis, and the results are shown in Fig. 13. 2DCOS can discern the specific order taking place under external perturbation. In this
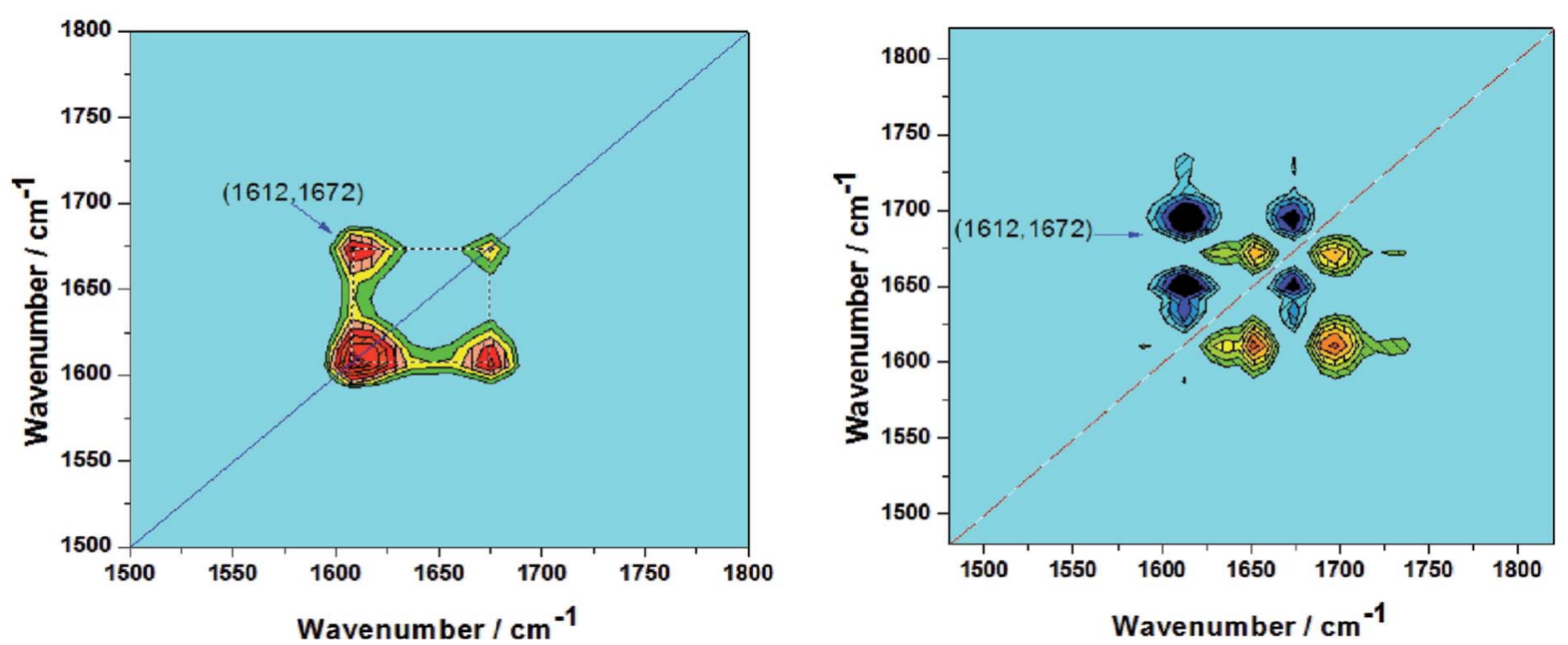

Fig. 13 Synchronous (left) and asynchronous (right) spectra of 2DCOS for carbonyl of the HPAM/UR composite solution. In the contour maps, warm colors are defined as positive intensities, whereas the cool colors are defined as negative intensities. 
measurement, temperature served as a perturbation to explore the effect on the infrared absorption peak in the HPAM/UR system. The synchronous and asynchronous spectra, which are located at the 1500-1800 wavenumber, for carbonyl absorption of the HPAM/UR blend solution are obtained by the $2 \mathrm{D}$ shige. The determining rule can be simply summarized as Noda's rule, that is, the cross-peaks $v_{1}$ and $v_{2}$ (assuming $v_{2}$ is less than $v_{1}$ ) in the synchronous and asynchronous spectra have the same sign, and it indicates that the variation of $v_{1}$ may occur before that of $v_{2}$, and vice versa. If the sign is zero or blank, an exact judgment cannot be made. In the synchronous spectra, two self-correlation peaks are located at the diagonal line with the wavenumber $1612 \mathrm{~cm}^{-1}$ and $1672 \mathrm{~cm}^{-1}$ corresponding to the absorbance of the carbonyl functional group, affected by UR and water through molecular hydrogen bonding, respectively. A positive cross-peak appears at (1612, 1672) $\mathrm{cm}^{-1}$ in the synchronous spectra, whereas in the asynchronous spectra, the sign of the cross-peak $(1612,1672) \mathrm{cm}^{-1}$ is negative. According to the Noda's rule, the positive crosspeak in the synchronous spectra and negative cross-peak in the asynchronous spectra demonstrate that the larger wavenumber will respond to external perturbation earlier than the smaller wavenumber. In other words, hydrogen bonding between carbonyl group on the HPAM lateral chain and water is damaged initially and then the segmental free carbonyl group forms hydrogen bonding with UR. This result confirms the conclusions shown in Fig. 12.

\section{Conclusions}

In this study, the rheological properties, in combination with the DLS, DSC, and 2DCOS technique, were employed to elucidate the shear rheological behavior of HPAM/UR in an aqueous solution and the interplay between HPAM and UR influenced by the temperature. The main conclusions are summarized as follows.

Due to the addition of UR, visible improvements in the viscoelastic properties of the HPAM aqueous solution were observed. Its intrinsic viscosity, hydraulic size, apparent viscosity, modulus, and first normal stress difference increase under the present measurement conditions. The UR content and temperature have great influence on the interaction mode and strength. The maximum value of the intrinsic viscosity occurred at the UR content of $300 \mathrm{mg} \mathrm{L}^{-1}$. The highest modulus and apparent viscosity for the HPAM/UR solution were observed when the temperature was in the vicinity of $45^{\circ} \mathrm{C}$. Based on the rheological data, it is found that HPAM/UR has better viscoelasticity performance that can be ascribed to a larger hydraulic size of HPAM in the aqueous solution. The DLS measurements indicate that the $2000 \mathrm{mg} \mathrm{L}^{-1} \mathrm{HPAM} / \mathrm{UR}$ solution has a distribution of the hydraulic diameter in the range 200-700 nm, which is larger than that of the HPAM solution. The stronger hydrogen bonding interaction exists between the carbonyl and amino groups in this system in the vicinity of $45{ }^{\circ} \mathrm{C}$, which is confirmed by DSC and 2DCOS techniques from a different perspective.

\section{Acknowledgements}

This work was supported by the Natural Science Foundation (Project No. Province 596102A071) from the Sichuan Provincial Educational Department of China and projects (material B026, new material 046) from Geological Research Institute of Shengli Oil Field, Sinopec. Ltd. Corp.

\section{References}

1 M. G. Zhang, L. H. Zhang and B. Jiang, Rheological properties of salt-tolerant HPAM solutions with ultrahigh molecular weight, J. Cent. South Univ. Technol., 2008, 15(s1), 93-97.

2 L. W. Meng, W. L. Kang and Y. Zhou, Viscoelastic rheological property of different types of polymer solutions for enhanced oil recovery, J. Cent. South Univ. Technol., 2008, 15(s1), 126129.

3 Z. Lei, C. W. Xiao and Y. Feng, Interfacial dilational properties of partly hydrolyzed polyacrylamide and gemini surfactant at the decane-water interface, Colloid Polym. Sci., 2008, 286(11), 1291-1297.

4 K. Prasuna, V. Gunjan and V. K. Aswal, Viscoelastic fluids originated from enhanced solubility of sodium laurate in cetyl trimethyl ammonium bromide micelles through cooperative self-assembly, J. Phys. Chem. B, 2010, 114, 10462-10470.

$5 \mathrm{~J}$. P. Yang and H. S. Li, The static adsorption of HPAM on porous media-Influence of hydrolysis degree on the quantity of adsorbed HPAM, Acta Polym. Sin., 1997, 5, 601605.

6 T. Krister, L. Bjolrn and N. Bo, Effect of hydrophobic modification of a nonionic cellulose derivative on the interaction with surfactants rheology, J. Phys. Chem. B, 1997, 101, 6450-6459.

7 Q. Zhang, J. S. Zhou, Y. A. Zhai, F. Q. Liu and G. Gao, Effect of salt solutions on chain structure of partially hydrolyzed polyacrylamide, J. Cent. South Univ. Technol., 2008, 15(s1), 080-083.

8 H. J. Gong, G. Y. Xu, Y. Y. Zhu, Y. J. Wang, D. Wu, M. Y. Niu, L. S. Wang, H. J. Guo and H. B. Wang, Influencing factors on the properties of complex systems consisting of hydrolyzed polyacrylamide/triton X-100/cetyl trimethyl ammonium Bromide: viscosity and dynamic interfacial tension studies, Energy Fuels, 2009, 23, 300-305.

9 M. Y. Khan, A. Samanta, K. Ojha and A. Mandal, Interaction between aqueous solutions of polymer and surfactant and its effect on physicochemical properties, Asia-Pac. J. Chem. Eng., 2008, 3, 579-585.

10 S. A. Frangou, E. R. Morris, D. A. Rees, R. K. Richardson and S. B. Ross-Murphy, Molecular origin of xanthan solution rheologv: Effect of urea on chain conformation and interactions, J. Polym. Sci., Part C: Polym. Lett., 1937, 20, 531-538.

$11 \mathrm{M}$. $\mathrm{Li}$ and $\mathrm{C}$. $\mathrm{Wu}$, Self-association of poly(Nisopropylacrylamide) and its complexation with gelatin in aqueous solution, Macromolecules, 1999, 32, 4311-4316. 
12 M. T. Ghannam, Rheological properties of aqueous polyacrylamide/NaCl solutions, J. Appl. Polym. Sci., 1999, 72, 1905-1912.

13 Q. Zhang, J. S. Zhou and Y. A. Zhai, Effect of salt solutions on chain structure of partially hydrolyzed polyacrylamide, $J$. Cent. South Univ. Technol., 2008, 15(s1), 80-83.

14 M. Y. Khan, A. Samanta and A. Mandal, Interaction between aqueous solutions of polymer and surfactant and its effect on physicochemical properties, Asia-Pac. J. Chem. Eng., 2008, 3, 579-585.

15 K. Thuresson, B. Lindman and B. Nystrom, Effect of hydrophobic modification of a nonionic cellulose derivative on the Interaction with surfactants rheology, $J$. Phys. Chem. B, 1997, 101, 6450-6459.

16 X. Xin, G. Y. Xu and H. G. Gong, Interaction between sodium oleate and partially hydrolyzed polyacrylamide: A rheological study, Colloids Surf., A, 2008, 321(1), 1-9.

$17 \mathrm{X}$. Xin, G. Y. Xu and D. Wu, The effect of $\mathrm{CaCl}_{2}$ on the interaction between hydrolyzed polyacrylamide and sodium stearate: rheological property study, Colloids Surf., A, 2007, 305, 138-144.

18 S. W. Cai, X. R. He, k. Liu, R. Zhang and L. Chen, Interaction between HPAM and urea in aqueous solution and rheological properties, Iran. Polym. J., 2015, 24, 663-670.

19 R. Sadeghi, S. Solaimani and N. Ebrahimi, Thermodynamic properties of anionic surfactant/polymer/water systems with respect to polymer-surfactant interactions and saltingeffect of surfactant on polymer in aqueous solutions, Fluid Phase Equilib., 2016, 425, 411-420.

$20 \mathrm{~J}$. Su, Q. Chu and M. Ren, Properties of high temperature resistance and salt tolerance drilling fluids incorporating acrylamide/2-Acrylamido -2-Methyl- 1-Propane sulfonic acid/ $N$-Vinylpyrrolidone/dimethyl diallyl ammonium chloride quadripolymer as fluid loss additives, J. Polym. Eng., 2016, 34(2), 153-159.

21 H. Cao, W. Y. Zhang and G. L. Zhao, Separation Method of an anoinic Surfactant from Crude Oil, Acta Sci. Nat. Univ. Jilin., 2000, 4, 88-90.

22 H. P. Xu, D. Y. Chen and S. Wang, Macromolecular selfassembly and nanotechnology in China, Philos. Trans. $R$. Soc., A, 2013, 9, 371.

23 Y. Li, L. Li and J. Q. Sun, Autonomic self-healing of hydrogel thin films, Angew. Chem., Int. Ed., 2010, 49, 767-771.

24 C. X. Luo, L. G. Zhang and J. X. Hou, Molecular weight mischaracterization of polyacrylamide for oil recovery by multiangle Light scattering, Acta Polym. Sin., 2012, 3, 313317.

25 L. J. Han, Z. B. Ye and H. Chen, Self-assembly of hydrophobically associating polyacrylamide and gemini surfactant, Acta Phys.-Chim. Sin., 2012, 28(6), 1405-1410.

$26 \mathrm{C}$. $\mathrm{Wu}$ and S. Q. Zhou, Laser light scattering study of the phase transition of poly( $N$-4-sopropylacrylamide $)$ in water: 1. Single Chain, Macromolecules, 1995, 28, 8381-8387.

27 J. P. Zhao, Amphiphilic/thermoresponsive block/graff copolymers: synthesis and aqueous solution properties, Master Thesis, University of Science and Technology, Shenzhen, China, 2010.
28 S. Q. Zhou and W. U. Chi, Laser light scattering characterization of the molecular weight distribution of poly( $N$-isoProPylacrylamide), Chem. J. Chin. Univ., 1994, 15, 1567-1571.

29 O. Oriekhova and S. Stoll, Effects of $\mathrm{pH}$ and fulvic acids concentration on the stability of fulvic acids - cerium (IV) oxide nanoparticle complexes, Chemosphere, 2016, 144, 131-137.

30 J. Kudr, L. Richtera, L. Nejdl, K. Xhaxhiu and P. Vitek, Improved electrochemical detection of zinc ions using electrode modified with electrochemically reduced graphene oxide, Materials, 2016, 9(31), 1-12.

31 P. E. Gibbs, T. Miralem, N. Lerner-Marmarosh and M. D. Maines, Nanoparticle delivered human biliverdin reductase-based peptide increases glucose uptake by activating IRK/Akt/GSK3 Axis: the peptide is effective in the cell and wild-type and diabetic $\mathrm{Ob} / \mathrm{Ob}$ mice, $J$. Diabetes Res., 2016, 2016(2), 1-15.

32 Y. Y. Bo, S. H. Moon, S. R. Seelam, J. J. Min and Y. S. Lee, Development of a multimodal imaging probe by encapsulating iron oxide nanoparticles with functionalized amphiphiles for lymph node imaging, Nanomedicine, 2016, 10(12), 1899-1910.

33 R. A. Nicodemus, K. Ramasesha, S. T. Roberts and A. Tokmakoff, Hydrogen bond rearrangements in water probed with temperature-dependent 2D IR, J. Phys. Chem. Lett., 2010, 1, 1068-1072.

34 K. Zheng, R. G. Liu and Y. Huang, A two-dimensional IR correlation spectroscopic study of the conformational changes in syndiotactic polypropylene during crystallization, Polym. J., 2010, 42, 81-85.

35 G. Tian, Q. Wu, S. Sun, I. Noda and G. Q. Chen, Studies on pre-melting and crystallization process of biosynthesized poly (3-hydroxybutyrate) using two-dimensional fouriertransform infrared spectroscopy, Chem. Res. Chin. Univ., 2002, 23, 1627-1631.

36 X. Y. Liu, T. Zhou and X. C. Wang, Investigation of selective molecular interactions using two-dimensional Fourier transform IR spectroscopy, Anal. Bioanal. Chem., 2010, 397, 339-343.

37 Q. Wang, W. Xu and P. Wu, New insights into the effects of Thermal treatment on the catalytic activity and conformational structure of glucose oxidase studied by electrochemistry, IR spectroscopy, and theoretical calculation, J. Phys. Chem. B, 2010, 114, 12754-12764.

38 I. Noda, Techniques useful in two-dimensional correlation and codistribution spectroscopy (2DCOS and 2DCDS) analyses, J. Mol. Struct., 2016, 1124, 29-41.

39 Z. Q. Zhang, J. Zuo and Y. Dan, Lacer light scattering study on solution behavior of poly(acrylamide-co-acrylic acid), Acta Polym. Sin., 2003, 8(4), 500-503.

40 C. X. Luo, G. Long and G. X. Hou, Molecular weight characterization of polyacrylamide for oil recovery by multiangle light scattering, Acta Polym. Sin., 2012, 3, 3-6.

41 X. R. He, S. W. Cai, H. Yu, Q. Chen and H. L. Hou, Differential spectrum applied in study of hydrogen bonding interaction in aqueous solution, Int. J. Polym. Anal. Charact., 2014, 19, 141-150. 
42 R. Zhang, X. R. He, S. W. Cai and K. Liu, Rheology of diluted and semi-diluted partially hydrolyzed polyacrylamide solutions under shear: Experimental studies, Petroleum, 2017, 3, 258-265.
43 X. R. He, R. Zhang, K. Liu, S. W. Cai and G. S. Huang, Rheological behaviors and molecular motions of semidiluted Xanthan solutions under shear: Experimental studies, Polym. Sci., Ser. A, 2014, 56, 687-696. 